\title{
Osteoarthritis of the hip conservative treatment with type A botulinum toxin
}

\author{
Sofía Durán-Hernández ${ }^{*}$, Juan Francisco Soto-Rodríguez², Jorge Allen-Bobadilla ${ }^{3}$, \\ Nayar Durán-Hernández ${ }^{1}$ and Pamela García-Mosco ${ }^{1}$ \\ ${ }^{1}$ Department of Physical Medicine and Rehabilitation; ${ }^{2}$ Department of Trauma and Orthopedics, Hospital General Tacuba, ISSSTE, ${ }^{3}$ Private practice; \\ City of Mexico, Mexico
}

\begin{abstract}
Introduction: Osteoarthritis $(O A)$ of the hip is a low-grade inflammatory disease of multiple etiology that has a limited conservative management and insufficiently explored. The application of botulinum toxin type A (BoNT-A) produces in the applied muscle a temporary, delimitable and reversible flaccid paralysis, which applied in a strategic way achieves to reduce mechanical stress and pain. Objective: To evaluate the effect on pain perception, functionality and rigidity and changes in flexibility, internal and external rotation, before and 90 days after the application of BoNT-A. Methods: Clinical, experimental, longitudinal, open study, with a total of 35 patients and 45 hips treated. BoNT-A of $500 \mathrm{U}$ was applied in iliac, adductor brevis and longus muscles. Results: The Wilcoxon test was used to compare the evaluation scores on days 0-90, observing the decrease in referred pain $(p<0.0001)$, rigidity $(p<0.002)$, improved perceived function $(p<0.001)$ and mobility arcs: flexion, internal and external rotation $(p<0.0001)$. Conclusions: The treatment with BoNT-A provides a conservative and safe option for the management of symptoms and physical restraint caused by hip $O A$.
\end{abstract}

KEY WORDS: Osteoarthritis. Botulinum toxin. Chronic pain.

\section{Introduction}

Osteoarthritis $(\mathrm{OA})$ is the most common arthropathy in the world. It affects synovial joints and causes destruction of articular cartilage, subchondral bone remodeling and inflammation of the synovial capsule and periarticular soft tissues. The most affected joints are the knee, hip and interphalangeal joints of the hands'.

Globally, it is considered the $13^{\text {th }}$ cause of years lived with disease ${ }^{2}$ and the $11^{\text {th }}$ cause of disability ${ }^{3}$. In the USA, prevalence increases with age, reaching $33.6 \%$ in adults older than 65 years $^{4}$. In Mexico, OA is the $8^{\text {th }}$ cause of family medicine appointments at the IMSS ${ }^{5}$ and has an estimated national prevalence of $10.51 \%$, which is higher in women $(11.7 \%)$ than in men $(8.71 \%)^{6}$.
The diagnosis of hip OA follows the 1991 American College of Rheumatology (ACR) guidelines and is addressed through history and physical examination, as well laboratory and radiographic examinations. In the algorithm, hip pain is taken into account plus two of the following criteria: 1) erythrocyte sedimentation rate $<20 \mathrm{~mm} / \mathrm{hour}, 2$ ) femoral or acetabular osteophytes and 3) narrowing of the upper, axial and/or medial joint space $^{7}$. OA severity assessment is carried out using the Kellgren and Lawrence radiological scale proposed in 1957, which considers four grades of severity with a progressive increase in joint compromise (Table 1).

OA was previously considered a degenerative disease and exclusive to articular cartilage but, currently, it is assumed as a chronic, low-grade inflammatory condition that fully affects the joint ${ }^{8}$. There are three main factors that participate in this process: the
Date of reception: 11-03-2019

Date of acceptance: 19-03-2019

DOI: 10.24875/GMM.M19000289
Gac Med Mex. 2019;155 (Suppl 1):S44-S48

Contents available at PubMed www.gacetamedicademexico.com 
Table 1. Kellgren and Lawrence radiological scale for hip OA

\begin{tabular}{lll}
\hline OA grade & Characteristics \\
\hline Grade I & (Doubtful OA) involves medial joint space narrowing and osteophytes around the femoral head \\
Grade II & (Moderate OA) frank joint space narrowing, well-defined osteophytes and slight sclerosis on the joint surface \\
Grade III & $\begin{array}{l}\text { (Moderate OA) marked joint space narrowing, osteophytes, subchondral sclerosis, cyst formation and femoral head and } \\
\text { acetabulum deformity }\end{array}$ \\
Grade IV & $\begin{array}{l}\text { (Severe OA) direct contact of joint surfaces, subchondral sclerosis, cysts, marked deformity of the femoral head and } \\
\text { acetabulum and large osteophytes }\end{array}$
\end{tabular}

inherent characteristics of the specialized cells that make up the joint, the mechanical stress they are subjected to and the effect the systemic environment exerts on them. In addition to participating as a damage recipient, innervation is an important inflammation regulator, since it modulates vascular permeability. This statement is based on the observation of patients with rheumatoid arthritis who suffered paresis or paralysis of the affected limb, whose symptoms were reduced or resolved in previously affected joints. This led to the reproduction of the phenomenon in animal models by producing inflammatory arthritis and subsequent surgical denervation. In these experiments, the expression of genes involved in vascular permeability and trans-endothelial cell migration was found to be altered, with inflammation reversal being achieved ${ }^{9}$.

The pre-surgical therapeutic options for hip OA were reviewed by the ACR in 2012. Inclusion of patients in a cardiovascular exercise (and weight loss if necessary) program. Regarding pharmacological measures, it is recommended that patients use analgesics p.r.n. and that these are selected in a gradient from lower to higher incidence of side effects: paracetamol, non-steroidal analgesics (NSAIDs), tramadol and intra-articular corticosteroid injection. It is important mentioning that, unlike other types of $O A$, this guideline does not recommend the use of chondroitin sulfate and glucosamine, topical NSAIDs or hyaluronate intra-articular injections ${ }^{10}$.

Non-prosthetic surgical treatments include partial or complete tenotomies of adductors and muscles inserted in the anterior iliac spine, which reduce muscle tension and mechanical stress between the acetabulum and the femoral head (Voss surgery). Given that botulinum toxin type A (BoNT-A) causes a chemical limited and transient denervation with subsequent flaccid paralysis of the injected striated muscle, which ultimately produces an effect that is similar to that of said technique by applying it only to the adductors and iliac portion of the iliac psoas ${ }^{11}$.
Botulinum toxin has seven different serotypes (A-G). The mechanism of action resides in its protease nature, which enables for it to cleave the synaptic transport proteins involved in neurotransmitter exocytosis. BoNT-A specifically acts cleaving the transport vesicle SNAP-25 (synaptosomal-associated $25 \mathrm{kDa}$ protein), which produces the secretion of acetylcholine. This selective effect is the reason for its therapeutic use in diseases characterized by cholinergic terminals hyperfunctionality ${ }^{12,13}$. Classically, this effect has been regarded as local, although recent research shows that the toxin can migrate trans-axonally from the synaptic terminal in a retrograde manner. An inhibitory effect on glutamate and substance $P$ secretion in spinal cord dorsal horn astrocytes has been reported, which could partially inhibit over-excitation in nociceptive transmission ${ }^{13}$.

In this context, the approach with BoNT-A was proposed in a condition of chronic pain associated with marked mechanical stress such as hip OA. Previously used in $\mathrm{OA}$ of the knee and hip in animals and humans, the toxin offers an innovative and safe option $^{14,15}$. The purpose of this study is to assess whether BoNT-A has an analgesic effect by reducing mechanical stress between the femoral head and the acetabulum, as well as on the regulation of pain-related neurotransmitters, such as substance $p$ and glutamate, and assess if there is a beneficial effect in the treatment of these patients during the time the effect of the toxin lasts ( 3 months).

\section{Methods and patients}

An experimental, longitudinal, open label clinical trial was carried out. Using a non-probabilistic sample, patients were recruited at the Physical Medicine and Rehabilitation clinic of the Tacuba General Hospital (ISSSTE) in the period from December 2016 to March 2017. Patients of legal age, from both genders, diagnosed with $O A$ according to ACR criteria and with 
severity grades II to IV according to the Kellgren and Lawrence scale and regardless of evolution time were included. Patients were excluded if they: a) had received an intra-articular injection within the previous 6 months; b) had conditions that might interfere with treatment efficacy (e.g. local, systemic infection); c) had medical conditions that contraindicated the use of therapy with BoNT-A (coagulopathies, pregnancy, breastfeeding, known allergy to the treatment), and d) had concomitant diseases that interfered with the correct analysis of the results (associated neuromuscular conditions, rheumatic diseases diagnosis).

\section{Ethical aspects}

The present study was conducted in accordance with the principles of the Declaration of Helsinki. The study protocol was subjected to evaluation by the Hospital Research Committee (HRC) and the Hospital Research Ethics Committee (HREC) of the Tacuba General Hospital (approval and registration number: 035-2016). It was subsequently registered and approved by the ISSSTE National Research Committee (registration number 498-2016). All patients were informed verbally and in writing about the details of the research protocol, their doubts were fully clarified and only those who signed the informed consent document were included. Treatment of their condition was by no means limited in case they refused to participate in the study.

\section{Study design}

Patients were assessed on day 0 and day 90. At each one of the two patient visits, the Western Ontario and McMaster Universities Arthritis Index (WOMAC) questionnaire was applied for pain (5 questions), stiffness (2 questions) and function assessment (17 questions), using a 0 to 4 Likert scale for each question. The higher the score, the higher the assumed level of symptomatic compromise and disability. In addition, a visual analogue scale (VAS) was used for assessment of pain during the 24 hours prior to the visit. Patient functional assessment was carried out with goniometry, with flexion, internal rotation and external rotation of the hip assessed by the same evaluator.

Following the therapeutic schemes proposed by the ACR, standard non-pharmacological treatment for hip OA was implemented, which consisted of 5 days of local heat therapy for 10 minutes, as well as teaching and subsequent execution of pelvic limb stretching
Table 2. Patient demographic data. Demographic variables mean and standard deviation are shown. The first section considers $\mathrm{n}=\mathbf{3 5}$ patients. The Kellgren and Lawrence radiological scale score contemplates a total of 45 hips

\begin{tabular}{|c|c|}
\hline \multicolumn{2}{|l|}{ Patient demographic data } \\
\hline $\begin{array}{l}\text { Age, years, mean (SD) } \\
\text { Range }\end{array}$ & $\begin{array}{c}n=35 \\
62.57(10.79) \\
42-84\end{array}$ \\
\hline Gender (females/males), n & $31 / 4$ \\
\hline $\begin{array}{l}\mathrm{BMI}, \mathrm{kg} / \mathrm{m}^{2} \text {, mean }(\mathrm{SD}) \\
\text { Range }\end{array}$ & $\begin{array}{l}29.14(3.61) \\
21.43-35.7\end{array}$ \\
\hline Bilateral/unilateral involvement, $\mathrm{n}$ & $10 / 25$ \\
\hline $\begin{array}{l}\text { Kellgren-Lawrence, n (\%) } \\
\text { Grade II } \\
\text { Grade III } \\
\text { Grade IV }\end{array}$ & $\begin{array}{c}n=45 \\
12(26.7) \\
18(40) \\
15(33.3)\end{array}$ \\
\hline
\end{tabular}

SD: standard deviation; BMI: body mass index.

exercises and strengthening of ankle-to-knee muscles, gluteus minimus, biceps femoris and gluteus mayor, as well as training in joint hygiene measures.

\section{BoNT-A intramuscular application}

\section{Medication}

Abobotulinum toxin type A (Dysport, IPSEN 500 IU) was diluted in $1 \mathrm{ml}$ of sterile saline. In all patients, a total of $350 \mathrm{IU}(0.7 \mathrm{ml})$ was applied to the iliac portion of the iliopsoas iliac muscle and $350 \mathrm{IU}$ to the adductoris brevis and adductoris longus muscles (175 IU or $0.35 \mathrm{~mL}$ each) with a $27 \mathrm{G}$ needle, of an inch and a half in length.

\section{Treatment application}

It is carried out by an expert in the application of the medication using an anatomical reference. The patient is placed in the supine position with aseptic and antiseptic techniques in areas to be infiltrated for the iliopsoas iliac muscle: palpation of the anterior superior iliac spine is performed, placing the needle $2 \mathrm{~cm}$ away towards the abdomen and directing the needle towards the inner border of the iliac bone, penetrating 2 to $3 \mathrm{~cm}$ trying to bump into it and injecting the medication. Adductoris brevis muscles: the tendon of origin, which goes from the lower border of the ischiopubic branch to the ischial tuberosity is located and distally measured 7 to $8 \mathrm{~cm}$ (or four fingers below the pubis). For the adductor longus, the needle is introduced 1 to $3 \mathrm{~cm}$ deep, and for the adductor brevis, 3 to $5 \mathrm{~cm}$ deep at the aforementioned doses. 
Table 3. Baseline and day 90 values in the assessment and physical examination scales $(n=45)$

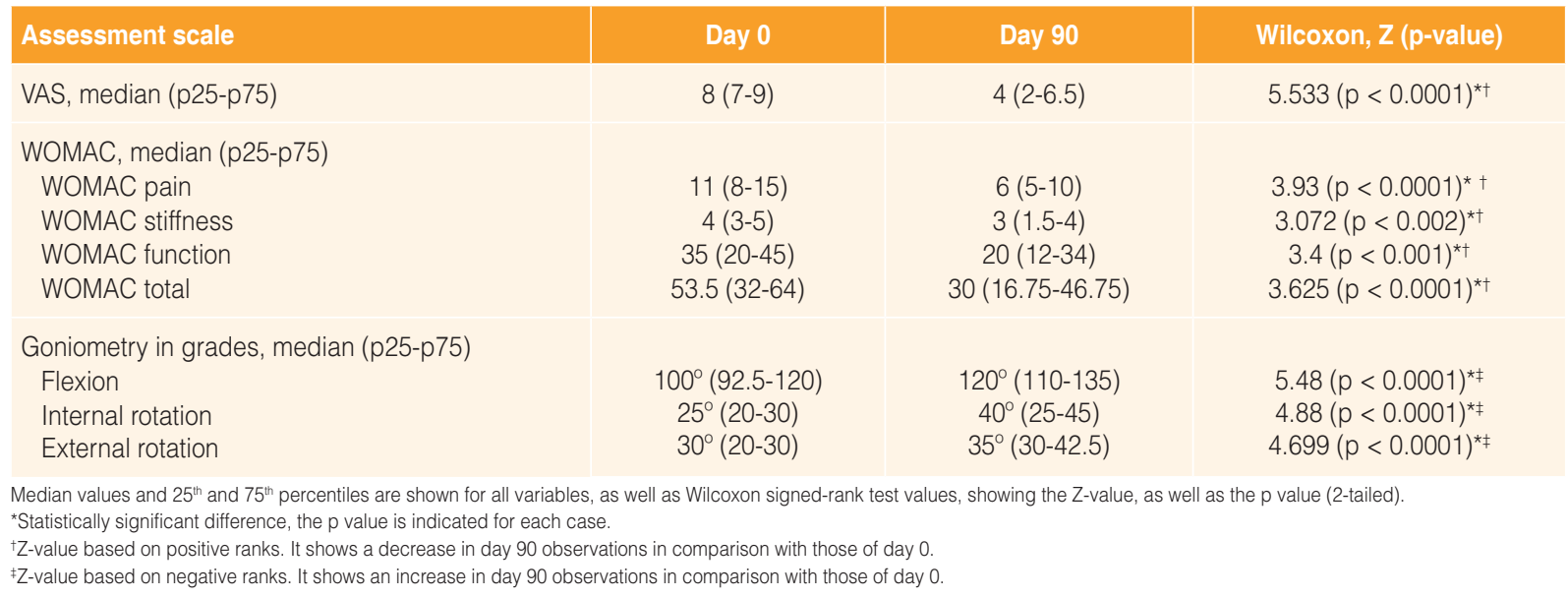

\section{Data analysis}

Data analysis was carried out in a blinded manner by a statistician unrelated to the patients or the study design. The statistical package SPSS ${ }^{\circledR}$ version 23 for MacOS (SPSS Inc., Chicago, IL, USA) was used. Descriptive statistics were used for demographic variables and assessed scales. Normality tests were performed for quantitative variables and when they did not have an adequate adjustment, non-parametric tests were used for their analysis. The measurements of variables at day 0 and 90 were compared with the Wilcoxon signed-rank test for paired samples. The results were considered statistically significant when the $p$-value was $<0.05$.

\section{Results}

A total of 39 patients were included, out of which 35 concluded the study. Ten patients with bilateral coxarthrosis were included, and thus a total of 45 hips is considered in the clinical variables. The patients were between the $5^{\text {th }}$ and $9^{\text {th }}$ decade of life, with a higher percentage of female patients $(88.57 \%)$. The average of patients had overweight. Severity grades of included hips were II (26.7\%), III (40\%) and IV (33.3\%) (Table 2).

There was a clear decrease in the VAS scores, and in each one of the WOMAC questionnaire sections and total score. Physical examination variables also showed a clear improvement, with an increase in the capacity of flexion, internal rotation and external rotation as measured by goniometry. In all cases, statistically significant differences were found (Table 3).

\section{Discussion}

BoNT-A is a medication that is widely used for dystonia and muscle spasticity in children and adults, which improves functionality, mobility and pain reduction, thus increasing quality of life ${ }^{16}$. Owing to this use, its influence was detected in the control of neuropathic pain in these patients and studies at the basic level that demonstrated the control of substance $P$ and glutamate, both related to SNAP-25 presynaptic release system ${ }^{17}$.

Although in the present study a joint compression release effect is achieved by relaxing some of the stabilizing muscles and temporarily changing the load axis, it should be noted that patients referred pain relief and increased ranges of immediate movement, possibly related to the previously-referred analgesic effect.

In 2010, Marchiani et al. published a study on the management of coxarthrosis with BoNT-A, where a significant decrease in referred pain and functional increase was achieved by applying a total of 400 units of BoNT-A to the adductor longus and magnus hip muscles. The sample in this study was 39 patients, and the VAS and the Short Form-36 Health Survey (SF-36) questionnaire were administered. However, no information on arthrometry was included, as it was done in the present study ${ }^{14}$.

The present study has the limitations of having an open-label design and lacking a control group due to the fact that it was a pilot study, the first one in Mexico and the second reported in the world. However, it adds evidence on the use of this treatment for the long-term control of chronic pain in hip OA.

The conservative management of this nosological entity often fails due to poor tolerance to exercises 
owing to pain, long transfers to get to the treatment and to the workplace and home, architectural barriers at workplace, home or even at the hospital level, which causes greater stress in the patient, exhaustion and treatment abandonment.

\section{Conclusions}

The application of BoNT-A to the iliac muscle and brevis and longus adductors decreases patient-referred pain and stiffness and increases the functionality perceived and found on physical examination. It is necessary to continue the study of botulinum toxin analgesic properties in studies with larger samples, of blinded and randomized design, and with the integration of more clinical variables, such as pre and post-treatment imaging control for a prolonged time.

\section{Conflict of interests}

The authors declare that they have no conflicts of interest.

\section{References}

1. National Clinical Guideline Centre. National Institute for Health and Clinical Excellence: Guidance. En: Osteoarthritis: Care and management in adults. Londres, Reino Unido: National Institute for Health and Care Excellence; 2014.

2. GBD 2016 Disease and Injury Incidence and Prevalence Collaborators. Global, regional, and national incidence, prevalence, and years lived with disability for 328 diseases and injuries for 195 countries, 1990-2016: a systematic analysis for the Global Burden of Disease Study 2016. Lancet. 2017;390(10100):1211-59.
3. Cross M, Smith E, Hoy D, Nolte S, Ackerman I, Fransen M, et al. The global burden of hip and knee osteoarthritis: estimates from the global burden of disease 2010 study. Ann Rheum Dis. 2014;73:1323-30.

4. Hootman JM, Helmick CG, Barbour KE, Theis KA, Boring MA. Updated projected prevalence of self-reported doctor-diagnosed arthritis and arthritis-attributable activity limitation among US adults, 2015-2040. Arthritis Rheumatol. 2016;68:1582-7.

5. Atención del paciente con osteoartritis de cadera y rodilla en el primer nivel. Secretaria de Salud Mexico [Internet]. México: gob.mx [fecha de última actualición: marzo de 2014]. Disponible en: https://cenetec-difusion.com/gpc-sns/?p=1287.

6. Pelaez-Ballestas I, Sanin LH, Moreno-Montoya J, Alvarez-Nemegyei J, Burgos-Vargas R, Garza-Elizondo M, et al. Epidemiology of the rheumatic diseases in Mexico. A study of 5 regions based on the COPCORD methodology. J Rheumatol. 2011;86:3-8.

7. Altman R, Alarcon G, Appelrouth D, Bloch D, Borenstein D, Brandt K, et al. The American College of Rheumatology criteria for the classification and reporting of osteoarthritis of the hip. Arthritis Rheum. 1991; 34:505-14.

8. Robinson WH, Lepus CM, Wang Q, Raghu H, Mao R, Lindstrom TM, et al. Low-grade inflammation as a key mediator of the pathogenesis of osteoarthritis. Nat Rev Rheumatol. 2016;12:580-92.

9. Ospelt C, Frank-Bertoncelj M. Why location matters - site-specific factors in rheumatic diseases. Nat Rev Rheumatol. 2017;13:433-42.

10. Hochberg MC, Altman RD, April KT, Benkhalti M, Guyatt G, McGowan J, et al. American College of Rheumatology 2012 recommendations for the use of nonpharmacologic and pharmacologic therapies in osteoarthritis of the hand, hip, and knee. Arthritis Care Res (Hoboken). 2012;64:465-74.

11. Kollberg G, Lundholm G. The Voss operation in osteoarthritis of the hip. Acta Orthop Scand. 1965;36:82-8.

12. Filipovic B, Matak I, Bach-Rojecky L, Lackovic Z. Central action of peripherally applied botulinum toxin type $A$ on pain and dural protein extravasation in rat model of trigeminal neuropathy. PLoS One. 2012;7:e29803.

13. Marinelli S, Vacca V, Ricordy R, Uggenti C, Tata AM, Luvisetto S, et al. The analgesic effect on neuropathic pain of retrogradely transported botulinum neurotoxin A involves Schwann cells and astrocytes. PLoS One. 2012;7:e47977.

14. Marchini C, Acler M, Bolognari MA, Causero A, Volpe D, Regis D, et al. Efficacy of botulinum toxin type A treatment of functional impairment of degenerative hip joint: Preliminary results. J Rehabil Med;42:691-3.

15. Boon AJ, Smith J, Dahm DL, Sorenson EJ, Larson DR, Fitz-Gibbon PD, et al. Efficacy of intra-articular botulinum toxin type $A$ in painful knee osteoarthritis: a pilot study. PM \& R: the journal of injury, function, and rehabilitation. 2010:2:268-76.

16. Cubillos O, Maldonado A, Moyano Vera Á, San Martín E. Toxina botulínica y su importancia en el campo de la rehabilitación. Rev Hosp Clín Univ Chile 2010; 21: 319 - 25.

17. Cuevas-Trisan R, Motta-Valencia KJ. Botulinum toxins for pain management: A comprehensive review. J Rehabil Med. 2013;1:51-64. 